\title{
A single session of music therapy decreased anxiety and improved relaxation in adults who required mechanical ventilation
}

\author{
Chlan L. Effectiveness of a music therapy intervention on relaxation and anxiety for patients receiving ventilatory assistance. \\ Heart Lung 1998 May/Jun;27:169-76.
}

\section{Question}

Does a single session of music therapy increase relaxation and reduce anxiety in adults who receive mechanical ventilation?

\section{Design}

Randomised controlled trial.

\section{Setting}

4 intensive care units of 3 urban university teaching hospitals in midwestern USA.

\section{Patients}

54 adults (age range 18-89 y, mean age 57 y, 92\% white, $59 \%$ women) who were in the intensive care unit and needed mechanical ventilation. Inclusion criteria were being alert and mentally competent, having adequate hearing, speaking English as the primary language, and not receiving continuous intravenous sedation. Follow up was $91 \%$.

\section{Intervention}

All patients were provided with a restful atmosphere for $30 \mathrm{~min}$ utes: blinds were closed, lights were dimmed, a "do not disturb" sign was put on the door of each patient's room, and patients were told to relax and to think of something pleasant. 27 patients were allocated to receive 30 minutes of music therapy using cassette tape players and headphones. Music contained no lyrics, was designed to be relaxing, and had 60-80 beats/minute.
Patients chose from classical, New Age, country and western, religious, and easy listening music. 27 patients received no music (rest alone group).

\section{Main outcome measures}

Self reported state anxiety (feelings of tension, apprehension, nervousness, and worry) was measured using the Spielberger State-Trait Anxiety Inventory at baseline and after treatment. Physiological relaxation was assessed using heart and respiratory rate changes over time.

\section{Main results}

Patients chose classical (56\%), country and western (28\%), easy listening (12\%), and New Age (4\%) music. Patients in the music group had a lower mean score for state anxiety than patients in the rest alone group (10.1 $v 16.2, \mathrm{p}<0.001)$, and also had a greater reduction over time in heart and respiratory rate $(\mathrm{p}<0.001$ for both).

\section{Conclusion}

A single 30 minute music session reduced anxiety and increased relaxation (reduced heart and respiratory rate) for adults in the intensive care unit who required mechanical ventilation.

Sources of funding: in part, Sigma Theta Tau, Zeta Chapter; National Institute of Nursing Research.

For correspondence: $\operatorname{Dr}$ L Chlan, College of Nursing, 101 Nursing Building, University of Iowa, Iowa City, IA 52242, USA. Fax +13193359990.

\section{Commentary}

Patient anxiety associated with mechanical ventilatory assistance is universally accepted as a substantial problem. Administration of pharmacological anxiolytic agents for anxiety states can cause untoward physiological and psychological side effects. ${ }^{12}$ Research into nonpharmacological treatment for anxiety states in patients who need mechanical ventilation is therefore highly pertinent.

Several authors have examined the beneficial effects of relaxation methods and music therapy for adults and newborns who are critically ill..$^{3-5}$ Chlan's research uniquely examines this concept with specific reference to patients who are dependent on a ventilator.

It is important to understand the complexity of independent variables and the extent to which they can affect heart and respiratory rates in patients who are ventilated. Chlan acknowledges that giving specific sedative and cardiovascular medi- cations may have affected the findings. However, no adjustment was made for potential influences such as changes in levels of ventilatory support or administration of physiotherapy before the trial. Patients in this study received diverse levels of ventilatory support, which may have influenced physiological variables. The number of patients with spontaneous ventilation among the groups is unknown, diminishing the ability to generalise the results.

The reliability with which the StateTrait Anxiety Inventory is able to quantify "self reported" anxiety for patients who are unable to communicate verbally is also questionable.

The decrease in the physiological markers of heart rate and respiratory rate shown by this study are comparable with other music therapy studies of patients who are critically ill. ${ }^{3}$ Further work is required to develop an anxiety measure that is reliable for adults who are dependent on ventilators. The beneficial physiological responses shown in this and other studies, ${ }^{3}$ however, advocate the use of music therapy as a relaxation adjunct for patients who are critically ill.

\section{Jane Hardcastle, RGN, BSc Senior Staff Nurse General Intensive Care Unit The Royal Infirmary of Edinburgh Edinburgh, UK}

1 Creasey J. Sedation scoring. Nurs Crit Care 1996:1:171-7.

2 Shelly M. Sedation in the ITU. Care of the Critically Ill 1998;14:85-8.

3 Guzzetta CE. Effects of relaxation and music therapy on patients who are in a coronary care unit with presumed myocardial infarction. Heart Lung 1989;18:609-16.

4 Henry LL. Music therapy: a nursing intervention for the control of pain and anxiety in the ICU: a review of the literature. Dimens Crit Care Nurs 1995;14:295-304.

5 O'Sullivan RJ. A musical road to recovery: music in intensive care. Intensive Care Nurs 1991;7:160-3. 